\title{
Perspective
}

\section{Non-communicable diseases care model \& its integration with the primary health care system in Norway}

\author{
D. A. K. Perera ${ }^{1 *}$ and B. E. Moen ${ }^{1}$, \\ ${ }^{1}$ University of Bergen, 5020 Bergen, Norway \\ *Corresponding author, (iD https://orcid.org/0000 000301458454
}

\begin{abstract}
Non-communicable diseases (NCDs) account for an estimated $71 \%$ of all deaths globally. In Norway, in $2015,71 \%$ of all deaths were due to cardiovascular diseases, cancers and neurological disorders. NCDs are mostly chronic diseases where individuals live a long period with the disease, thus the disease becomes a part of everyday life. This perspective explores the NCD care model within the Norwegian health delivery system in relation to the integration of prevention, diagnosis, treatment and rehabilitation services of non-communicable diseases to the primary health care system, and also explores several best practices and lessons that can be adapted to minimize the critical gaps and areas of the non-communicable diseases care model in Sri Lanka.
\end{abstract}

Keywords: Primary health care system, General practitioners, Chronic diseases, personalized care, Continuity of care

Copyright: D.A.K. Perera and B.E. Moen, 2020. (c) (i) Commons Attribution License, which permits unrestricted use, distribution, and reproduction in any medium provided the original work is properly cited.

Funding: None

Competing interests: None

Received: 19 June 2020

Published: 31 December 2020

$\bowtie$ Correspondence: sjwprr@yahoo.com

Cite this article as: Perera DAK and Moen BE. Non-communicable diseases care model \& its integration with the primary health care system in Norway. Anuradhapura Medical Journal 2020;14 (2): 1-4.

DOI: http://doi.org/10.4038/amj.v14i2.7657

Norway is a high-income country in the Scandinavian region with a 5.1 million population. Its higher per capita expenditure in health has ensured one of the best health care systems with remarkable health status among the people. When considering the health system, the primary care sector is responsible for the provision of most health care needs of the population.

Non-communicable diseases (NCDs) cause 41 million deaths each year and account for an estimated $71 \%$ of all deaths globally [1]. In Norway, the three main cause-of-death disease groups cardiovascular diseases, cancers, and neurological disorders (mainly dementia) - contributed to $71 \%$ of all deaths in 2015 [2]. Tobacco, unhealthy diets, physical inactivity, and overconsumption of alcohol are the leading risk factors for most NCDs. NCDs are mostly chronic diseases where individuals live a long period with the disease; thus, the disease becomes a part of everyday life. Therefore, patients with NCDs need personalized care that extends beyond the usual medical treatment as well as care at the community and family level. Primary-healthcare facilities are patients' first point of contact with health services and hence the most appropriate places for patient screening and early disease detection, continuous care provision for uncomplicated patients, and referral of patients to specialists [3]. Griffin and Kinmonth (1998) say, 
General Practitioners (GPs) care of diabetic patients was better than that of specialists in hospitals; in such situations, patients of GPs had lower mortality rates and better glycemic control than did patients treated by specialists [4]. Most patients with NCDs do not require specialized care and can be managed appropriately at the primary care level, using simplified standard protocols supported by low-cost drugs and with links to higher levels of care for people who need them [3].

The main objective of this paper was to describe the NCD care model within the Norwegian health delivery system. Furthermore, this paper further explores the integration of prevention, diagnosis, treatment, and rehabilitation services of NCDs to the primary health care system within the Norwegian health system.

Data and information were gathered by reviewing national action plans, strategic plans, guidelines, regulations for the prevention and control of NCDs, and an extensive literature survey. In addition, several rounds of discussions and informal interviews with relevant officials were carried out. To get an in-depth understanding of the Norwegian NCD care model, several study visits and observations were performed.

\section{NCD care model in Norway}

Table 1: An overview of the Norwegian non-communicable disease (NCD) care model.

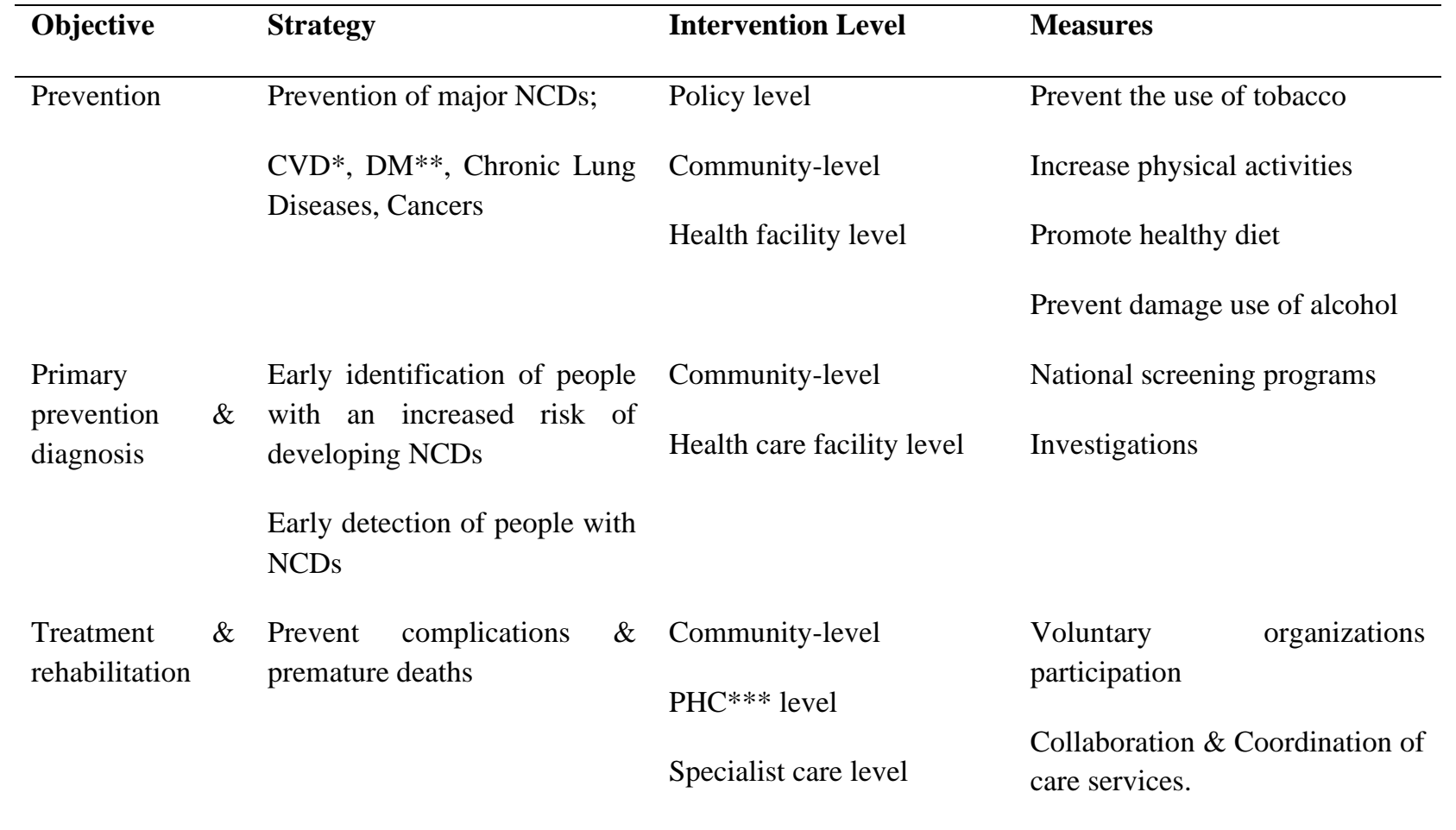

*Cardiovascular Disease **Diabetes Mellitus ***Primary Health Care

The Norwegian policy of health services provision is based on patients receiving the services they need at the most appropriate and efficient level [6]. In Norway, health service provision to the population is organized in two tiers as specialist health care and primary health care (PHC). Specialist health care services are delivered through a government-owned hospital network. The responsibility of delivering PHC services to the population was transferred to the municipalities or Kommunes by the 
Municipality Primary Healthcare Act introduced in 1984. PHC is administered by the municipality at the municipality level and is predominantly publicly funded with various copayments applicable.

GPs Care is the major element in the PHC system and works as the 'Gate Keepers' for specialist care. The NCDs prevention and control model is based on the GPs care. In 2018, there were 4217 GPs (79.5 GPs/100 000 population) practising in Norway [7].

GPs are responsible for making the diagnosis in NCDs except for cancers. They provide basic mental health care to the people. NCD care strategy is based on the principles of continuing and developing the primary preventive activities, early diagnosis, good quality at all stages of treatment and follow-up to secondary prevention, and reinforcing the roles of users and patients and getting them more actively involved in the treatment of their diseases [5]. The uniformity and standard of care have been ensured by the national clinical guidelines in NCD management available in the Norwegian electronic medical handbook for GPs. A diabetic patient visits the GP twice a year; one visit for cardiovascular assessment and the other one is for eye and foot assessment, and a hypertension patient does only one visit unless there are complications. Patients do blood sugar and pressure monitoring at home, and if need contact the GP. Annual health checkup provided by GP is an important element of the Norwegian NCD care model to prevent complications.

In addition to continuity of care, GP facilitates the coordination of care between specialist care and municipality as well as within and between the municipalities. The background to the Coordination Reform was the recognition that the structure of the health services has not sufficiently been adapted for patients with chronic diseases [5]. The Electronic Health Record (EHR) system has substantially contributed to improving the continuity and coordination of NCD care. Patients' health records can be accessed by different caregivers through the EHR system.

There is a national screening program for breast cancers (Mammogram), cervical cancers (Pap smear), and colorectal cancers. Healthy lifestyle centres (HLCs) and corresponding services have been established within the local authority level to improve primary prevention and early diagnosis. An HLC is an interdisciplinary primary health care service that offers effective, knowledge-based measures for people with or at high risk of the disease who need support in health behaviour change and in coping with health problems and chronic disease [8]. HLCs are mainly focused on preventing NCDs through the elimination of risk factors by promoting physical activities, healthy nutrition and tobacco cessation. Persons of all ages with a high risk of contracting a disease or who are already living with a disease and need help to change their living habits and manage their condition are eligible to attend HLCs. In 2013, HLCs had over 16 000 users [7].

The Norwegian health system encourages the participation of voluntary organizations in NCD care, especially in prevention and palliative works [9].

The main challenges of the Norwegian NCD care model are: health services place great emphasis on diagnosing and treating disease and major complications, and not enough on promoting health and preventing health problems, and many patients deteriorate and require hospital admission [5]. According to the GPs interviewed, lack of regular visits to the GP for NCD care, poor compliance, and poor risk factor modifications are the main factors for the patients' deterioration. However, evidence shows the PHC model is the most efficient and effective approach in the prevention and management of NCDs.

In Sri Lanka, although nearly 1000 primary curative care health facilities are in the country, about $60 \%$ of the clinic users for chronic diseases go to the secondary and tertiary care facilities [10]. Besides, mental health care is mostly provided by secondary and tertiary care facilities. This paper reveals several best practices and lessons that can be adapted to minimize the critical gaps and areas of the NCD care model in Sri Lanka; integrate NCD care into the PHC system, gatekeeping function at primary care level, continuity, and coordination of care within and between different care providers and national clinical guidelines in NCDs management for the primary care medical practitioners. 


\section{References:}

1. Non-communicable diseases, World Health Organization; 2018. Available from: http://www.who.int/en/news-room/fact-sheets/detail/noncommunicable-diseases.

2. Health Status in Norway 2017; Public health report, short version: NIPH, Norway, 2017.

3. Amarchand R, Krishnan A, Saraf DS, Mathur P, Shukla DK, Nath LM. Lessons for addressing noncommunicable diseases within a primary healthcare system from the Ballabgarh project, India. WHO South-East Asia J Public Health. 2015;4 (2):130-8. doi: 10.4103/2224-3151.206682.

4. Starfield B, Shi L, Macinko J. Contribution of primary care to health systems and health. Milbank Q. 2005;83(3):457-502. doi: 10.1111/j.1468-0009.2005.00409.x.

5. NCD strategy 2013 - 2017, Norway: https://www.regjeringen.no.

6. The Norwegian National Health Plan 2020- 2023; Ministry of health \& care services, Norway, 2019.

7. Statistics, Norway: https://www.ssb.no/en.

8. The Norwegian Health Directorate, 2019; https://www.helsedirektoratet.no

9. Støre GH, Minister of Health and Care Services, Norway; NCD strategy, 2013.

10. Annual Health Statistics; Ministry of Health, Nutrition and Indigenous Medicine - Sri Lanka, 2016. 